\title{
Seasonal distribution of attacks in aquaporin-4 antibody disease and myelin-oligodendrocyte antibody disease
}

\author{
Giordani Rodrigues dos Passos ${ }^{\mathrm{a}, 1}$, Liene Elsone ${ }^{\mathrm{b}, 1}$, Sebastian Luppe ${ }^{\mathrm{c}}$, Joanna Kitley ${ }^{\mathrm{a}}$, Silvia Messina ${ }^{\mathrm{a}}$, \\ Pedro María Rodríguez Cruz ${ }^{\mathrm{a}}$, Katharine Harding ${ }^{\mathrm{c}}$, Kerry Mutch ${ }^{\mathrm{b}}$, Maria Isabel Leite ${ }^{\mathrm{a}}$, Neil Robertson ${ }^{\mathrm{c}}$, \\ Anu Jacob $^{\mathrm{b}, 1}$, Jacqueline Palace ${ }^{\mathrm{a}, 1, *}$
}

${ }^{a}$ Nuffield Department of Clinical Neurosciences, John Radcliffe Hospital, University of Oxford, Oxford, United Kingdom

${ }^{\mathrm{b}}$ Department of Neurology, The Walton Centre NHS Foundation Trust, Liverpool, United Kingdom

${ }^{\mathrm{c}}$ Department of Neurology, University Hospital of Wales, Cardiff University, Cardiff, United Kingdom

\section{A B S T R A C T}

Background: Seasonal variation in incidence and exacerbations has been reported for neuroinflammatory conditions such as multiple sclerosis and acute disseminated encephalomyelitis (ADEM). It is unknown whether seasonality also influences aquaporin-4 antibody (AQP4-Ab) disease and myelin-oligodendrocyte antibody (MOG-Ab) disease.

Objective: We examined the seasonal distribution of attacks in AQP4-Ab disease and MOG-Ab disease.

Methods: Observational study using data prospectively recorded from three cohorts in the United Kingdom.

Results: There was no clear seasonal variation in AQP4-Ab or MOG-Ab attacks for either the onset attack nor subsequent relapses. In both groups, the proportion of attacks manifesting with each of the main phenotypes (optic neuritis, transverse myelitis, ADEM/ADEM-like) appeared stable across the year. This study is the first to examine seasonal distribution of MOG-Ab attacks and the largest in AQP4-Ab disease so far.

Conclusion: Lack of seasonal distribution in AQP4-Ab and MOG-Ab disease may argue against environment factors playing a role in the aetiopathogenesis of these conditions.

\section{Introduction}

Antibodies against aquaporin-4 (AQP4-Ab) and myelin-oligodendrocyte glycoprotein (MOG-Ab) are now recognised to cause neuromyelitis optica spectrum disorders (NMOSD), acute disseminated encephalomyelitis (ADEM) and, more rarely, with MOG-Ab, cortical encephalitis [1-3]. These antibodies are believed to be pathogenic, causing a primary astrocytopathy (AQP4-Ab) and a primary demyelinating disorder (MOG-Ab) [4,5]. Despite major advances in the understanding of their pathophysiology, putative triggers of autoimmunity in these conditions are yet to be identified.

Seasonal variation in manifestations of disease may reflect changes in environmental factors, some of which hold the potential to trigger or modulate biological processes involved in autoimmunity [6]. If present, a seasonal pattern may aid in the identification of such environmental factors [7]; furthermore, if not taken into account in clinics and research, it may bias longitudinal assessment of disease activity both at individual and group level $[8,9]$.
Unlike multiple sclerosis (MS), another immune-mediated, neuroinflammatory disease, wherein season of birth is a risk factor [10] and the incidence of relapses peaks in spring and early summer $[8,11,12]$, it is unknown whether seasonal variation exists in AQP4-Ab disease and MOG-Ab disease. We therefore aimed to examine the seasonal distribution of attacks in AQP4-Ab disease and MOG-Ab disease, looking at both total attacks and onset events within each group.

\section{Methods}

\subsection{Source of data}

We used data from three centres in the United Kingdom: the Walton Centre (Liverpool), the University Hospital of Wales (Cardiff) and the John Radcliffe Hospital (Oxford). Patients followed up in these cohorts had their clinical data prospectively recorded on each centre's database. Anonymised data relevant for this study was then retrieved from the local databases using a structured form.

\footnotetext{
* Corresponding author at: Nuffield Department of Clinical Neurosciences, Level 3 West Wing, John Radcliffe Hospital, Oxford OX3 9DU, United Kingdom.

E-mail address: jacqueline.palace@ndcn.ox.ac.uk (J. Palace)

1 These authors contributed equally to this work.
} 


\subsection{Population}

We included AQP4-Ab-seropositive patients and MOG-Ab-seropositive patients where there were exact attack dates recorded. All AQP4-Ab and MOG-Ab tests had been performed at the John Radcliffe Hospital using validated cell-based assays [13].

\subsection{Data collection and processing}

Basic demographic information and dates of the onset attack and subsequent relapses were obtained. For the Oxford cohort, information on the phenotype of attacks was also available. Only confirmed attacks whose start date was accurate at least to the month level were included in the analyses.

\subsection{Seasonality analysis}

A possible seasonal pattern was examined in two steps. Firstly, the observed frequency of attacks was computed by aggregating all attacks recorded over the years to monthly totals, whereas the expected frequency for each month was calculated assuming the null hypothesis of a uniform distribution across the year and taking into account the variable month lengths. Microsoft Excel(C) 2013 was used to produce radar plots (comparing the observed and expected monthly frequencies) and histograms (with observed frequencies normalised for a standard month duration of 30 days). These graphs were visually inspected for any recognisable pattern of seasonal variation. A similar procedure was done for the seasons, defined as follows: winter (December to February), spring (March to May), summer (June to August) and autumn (September to November). Secondly, considering that seasonal patterns may not be apparent upon visual inspection and periods of excess or reduction may not coincide with calendar-defined seasons [14,15], we used statistics to examine the monthly distribution of attacks. Since previously published data were insufficient and graphical inspection of our data did not permit clear a priori hypotheses regarding the specific patterns of seasonal distribution, the Freedman's test (Kuiper's $\mathrm{V}_{\mathrm{N}}$ statistic adapted for monthly grouped data) was selected, because it allows the detection of any deviation from a uniform seasonal distribution $[16,17]$. With the significance level set at $p<.05$, a sample size of at least 500 attacks would be required in each analysis to achieve $80 \%$ power in the detection of an annual sinusoidal curve with amplitude 0.2 (i.e. $40 \%$ difference between peak and trough) [18]. All analyses were done using DESCRIBEC, part of the WINPEPIC software package [19], version 11.65.

\subsection{Ethics approval}

All patients provided written consent to the use of their clinical data for research (Oxford Research Ethics Committee reference 16/SC/0224).

\section{Results}

We included 244 patients with AQP4-Ab-positive disease and 98 patients with MOG-Ab disease, for whom a total of 1000 and 235 confirmed attacks, respectively, had been recorded, of which 905 and 216 attacks, respectively, had accurate start date at least to the month level. Of these, 194 attacks in the AQP4-Ab group and 81 attacks in the MOG-Ab group were onset events. Further demographic and clinical data are shown in Table 1.

Monthly frequencies of attacks in each group are shown in Supplementary Table S1 (Supplemental data). The distribution of AQP4-Ab attacks across the months and seasons was remarkably similar to that expected, suggesting no seasonal variation (Fig. 1A and Fig. S1A-
Table 1

Demographic and clinical features of patients and attacks.

\begin{tabular}{|c|c|c|}
\hline & AQP4-Ab disease & MOG-Ab disease \\
\hline Patients, n & 244 & 98 \\
\hline Female, n (\%) & $204(83.6 \%)$ & $62(63.3 \%)$ \\
\hline Age at onset, median (range) & $43.1(2.7-84.0)$ & $27.8(2.5-69.4)$ \\
\hline Follow-up time ${ }^{a}$, median (range) & $\begin{array}{l}\text { years } \\
73.0(4.0-459.0) \\
\text { months }\end{array}$ & $\begin{array}{l}\text { years } \\
25.5(0.0-484.0) \\
\text { months }\end{array}$ \\
\hline $\begin{array}{l}\text { Attacks included in the analysis, } n \\
\text { of which: onset attacks, } n \\
\text { Attacks including the following } \\
\text { phenotypes }{ }^{b} \text { : }\end{array}$ & $\begin{array}{l}905 \\
194\end{array}$ & $\begin{array}{l}216 \\
81\end{array}$ \\
\hline $\mathrm{ON}, \mathrm{n}(\%)$ & $155(34.4 \%)$ & $151(69.9 \%)$ \\
\hline $\mathrm{TM}, \mathrm{n}(\%)$ & $278(61.8 \%)$ & $65(30.1 \%)$ \\
\hline ADEM or ADEM-like ${ }^{c}, \mathrm{n}(\%)$ & $65(14.4 \%)$ & $29(13.4 \%)$ \\
\hline
\end{tabular}

Abbreviations: AQP4-Ab = aquaporin-4 antibody; MOG-Ab = myelin-oligodendrocyte glycoprotein antibody; $\mathrm{n}=$ number; $\mathrm{ON}=$ optic neuritis; $\mathrm{TM}=$ transverse myelitis; ADEM $=$ acute disseminated encephalomyelitis.

a Based on the subset of patients for whom this information was available ( $n=180$ for AQP4-Ab disease and $n=98$ for MOG-Ab disease).

b Based on the subset of attacks for which this information was available ( $n=450$ for AQP4-Ab disease and $n=216$ for MOG-Ab disease). The sum within each group may exceed $100 \%$ due to attacks presenting with mixed phenotypes.

c The ADEM-like phenotype refers to involvement of the brain, brainstem or cerebellum, without meeting the usual definition of ADEM.

B), which was confirmed by adequately powered statistical analysis. When looking specifically at AQP4-Ab onset attacks, there seemed to be a slight, non-significant excess between late spring and summer (Fig. 1B and Fig. S1A-B).

MOG-Ab attacks were, overall, slightly more frequent in the winter, whereas onset attacks had possible peaks in April and June (Fig. 1C-D and Fig. S1C-D), which were not statistically significant in the seasonality test.

Analysis of seasonal distribution of attack phenotype (optic neuritis [ON], transverse myelitis [TM], ADEM/ADEM-like) in the Oxford cohort revealed no seasonal pattern in either MOG-Ab or AQP4-Ab disease (Fig. 2).

\section{Discussion}

Seasonal patterns in autoimmune diseases can be mediated by a number of environmental factors. Several infections and some vaccines have been linked to autoimmune conditions, including ADEM [20,21]. Night length and exposure to ultraviolet radiation modulate the secretion of melatonin and vitamin D, which exert immunomodulatory functions in MS and other immune-mediated diseases [6]. Indeed, counts of immune cells and levels of cytokines and chemokines have been shown to vary according to the season [22-24].

In particular, the role of vitamin D levels as a key factor in the seasonal variation in MS has been extensively studied. Vitamin D insufficiency is common in many regions worldwide, even in sunny, lower latitude areas, and has been associated with higher risk of developing MS, as well as higher disease activity and worse prognosis in patients with MS [6,25]. Vitamin D levels in NMOSD are less well studied, but may also influence disease severity [26], whereas the influence of vitamin D on MOG-Ab disease remains undetermined.

Studies from Japan and Brazil have not detected any significant seasonal variation in the incidence of NMOSD attacks $[27,28]$. These relatively small studies (with 131 and 282 attacks, respectively) have included both AQP4-Ab-positive and -negative cases, with the latter representing a somewhat heterogeneous group that may have encompassed other conditions and thus hindered the detection of a possible seasonal pattern. Furthermore, these studies have not looked specifically at onset attacks. To our knowledge, there are no previous stud- 
A AQP4-Ab (all attacks)

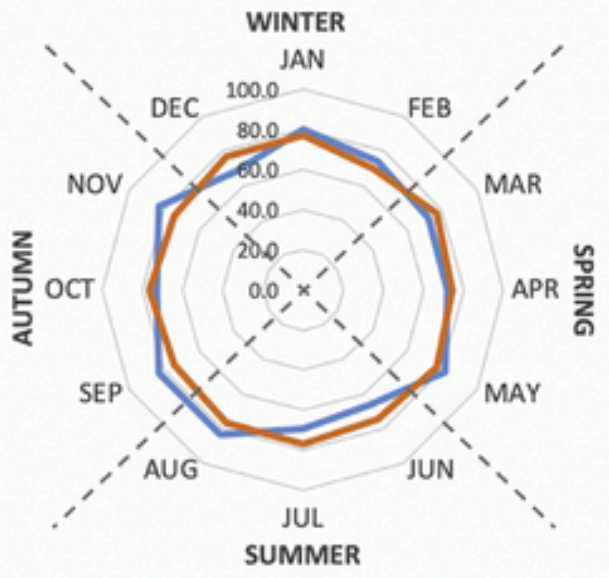

$V_{N}=0.022, p>0.1$

C

MOG-Ab (all attacks)

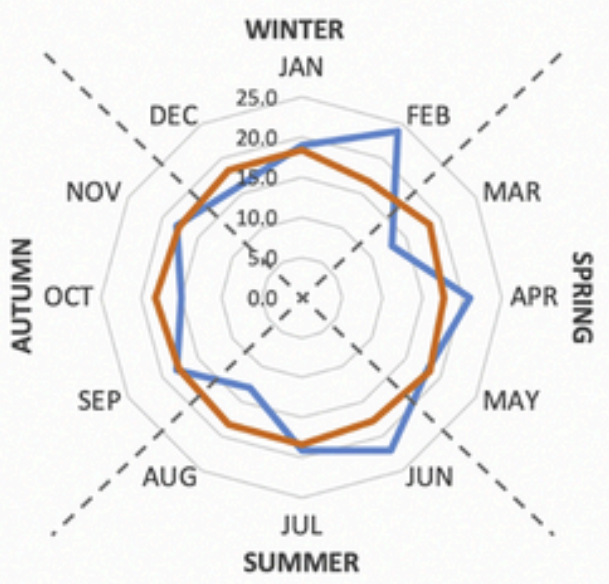

$V_{N}=0.049, p>0.1$
B

AQP4-Ab (onset attacks)

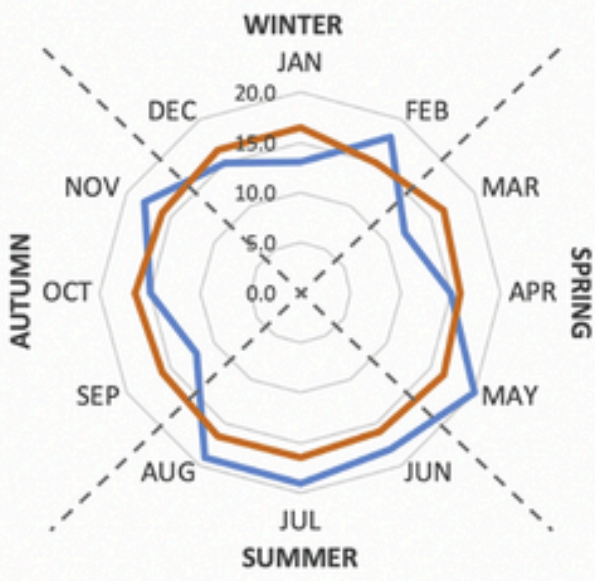

$V_{N}=0.055, p>0.1$

D

MOG-Ab (onset attacks)

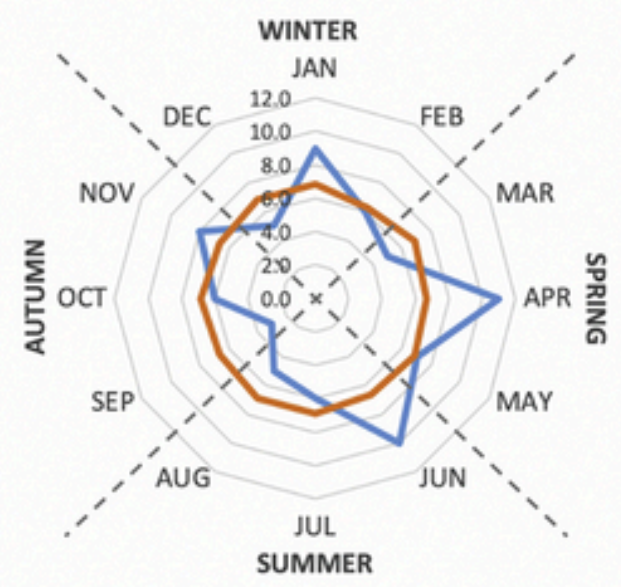

$V_{N}=0.097, p>0.1$

Fig. 1. Comparison between the observed and expected distribution of attacks across the year.

ies on seasonal patterns in MOG-Ab disease. On the other hand, there are several studies reporting a peak in the incidence of ADEM in winter and spring $[20,21,29]$, but these are older studies, published when testing for AQP4-Ab or MOG-Ab was not yet available. In the present study, no excess of ADEM relapses during spring or winter was noted in any of the groups.

The strengths of this study are the larger number of AQP4-Ab attacks than in previous seasonality studies, the addition of MOG-Ab attacks and the exploration of onset versus subsequent attacks as well as different attack types. In addition, we have performed statistical analyses appropriate for seasonality. Nonetheless, the number of attacks included is still relatively small, due to the rarity of these conditions, and seasonality tests usually have low power to detect patterns other than a simple sinusoidal curve with one peak and one trough six months apart [18]. Yet, previous MS studies using a comparable sample size were able to detect a seasonal effect [30-32].

\section{Conclusion}

There does not seem to be an important seasonal variation in AQP4-Ab disease attacks. A seasonal effect in MOG-Ab disease was not identified, but cannot be ruled out given the smaller sample size. In comparison to MS, these antibody-mediated diseases may be either less susceptible to environmental factors or susceptible to different ones with no seasonal variation.

Supplementary data to this article can be found online at https://doi. org/10.1016/j.jns.2020.116881. 
A

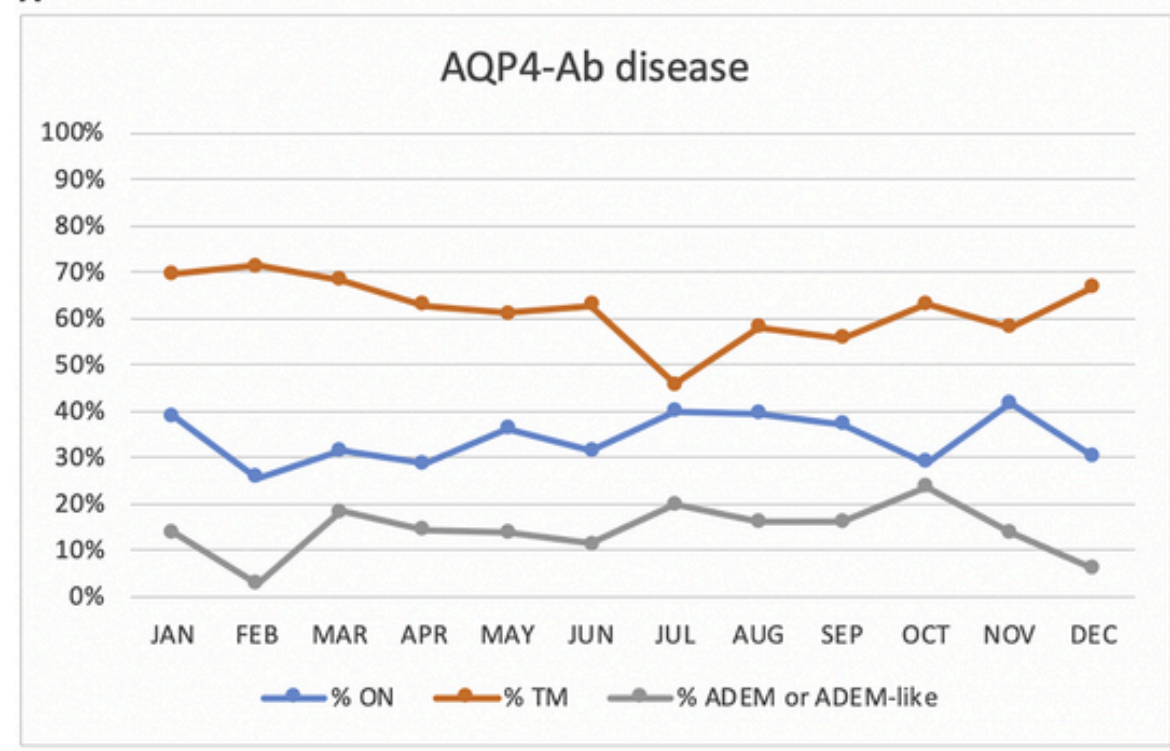

B

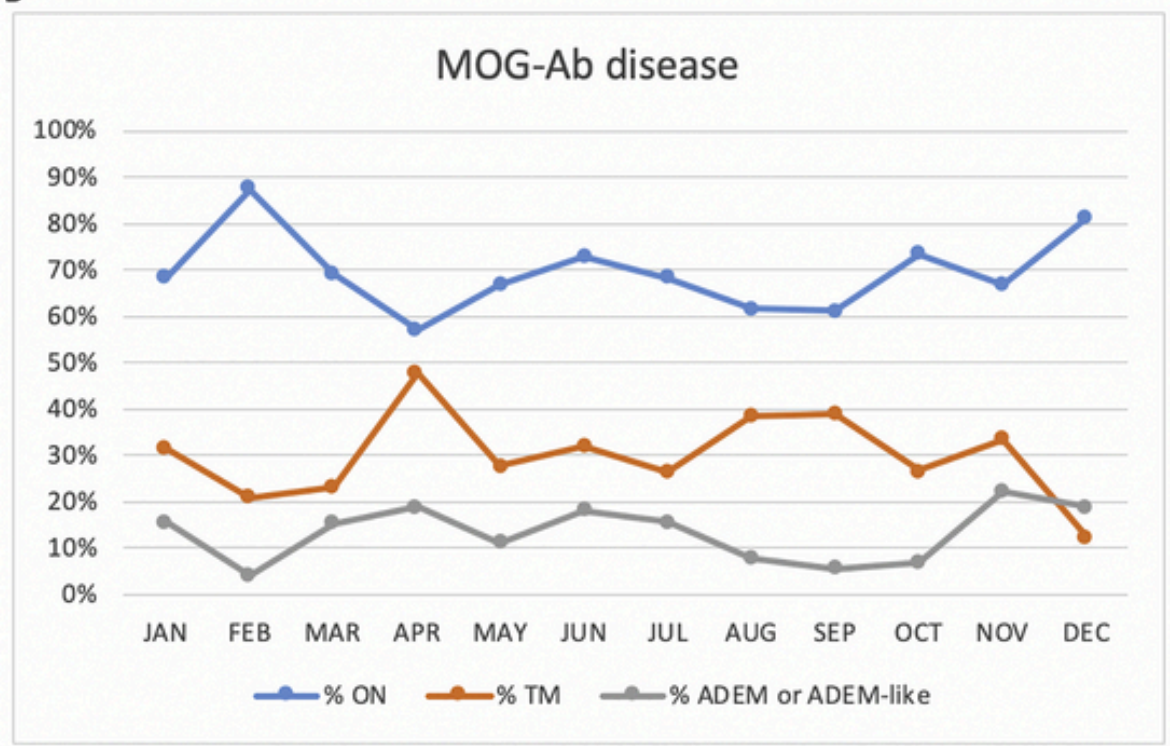

Fig. 2. Proportion of attacks presenting with each phenotype throughout the year.

Funding

No specific funding was needed for this study.

\section{Ethics approval}

All patients provided written consent to the use of their clinical data for research (Oxford Research Ethics Committee reference 16/SC/0224).

\section{Consent to participate}

Not applicable.

\section{Consent for publication}

Not applicable.

\section{Availability of data and material}

Not applicable.

\section{Code availability}

Not applicable.

\section{Authors' contributions}

Dr. dos Passos: conception of the work; data collection; data analysis and interpretation; drafting the article; critical revision of the article; final approval of the version to be published.

Dr. Elsone: conception of the work; data collection; data analysis and interpretation; drafting the article; critical revision of the article; final approval of the version to be published.

Dr. Luppe: data collection; critical revision of the article; final approval of the version to be published.

Dr. Kitley: data collection; critical revision of the article; final approval of the version to be published.

Dr. Messina: data collection; critical revision of the article; final approval of the version to be published. 
Dr. Rodríguez Cruz: data analysis and interpretation; critical revision of the article; final approval of the version to be published.

Dr. Harding: data collection; critical revision of the article; final approval of the version to be published.

Dr. Mutch: data collection; critical revision of the article; final approval of the version to be published.

Dr. Leite: conception of the work; critical revision of the article; final approval of the version to be published.

Dr. Robertson: conception of the work; critical revision of the article; final approval of the version to be published.

Dr. Jacob: conception of the work; data analysis and interpretation; drafting the article; critical revision of the article; final approval of the version to be published.

Dr. Palace: conception of the work; data analysis and interpretation; drafting the article; critical revision of the article; final approval of the version to be published.

The orange lines represent the expected number of attacks in each month (assuming there were no seasonal variation and taking into account the variable month lengths in days), while the blue lines represent the observed number of attacks. Large deviations of the blue lines from the orange lines indicate possible departures from a uniform seasonal distribution. The $\mathrm{V}_{\mathrm{N}}$ statistic and the corresponding $p$ value are based on the Freedman's test for monthly distribution of attacks in each group. Abbreviations: AQP4-Ab = aquaporin-4 antibody; MOG-Ab = myelin-oligodendrocyte glycoprotein antibody

Proportion of attacks presenting with each of three main phenotypes across the months. The sum of proportions in each month may exceed $100 \%$ due to attacks with mixed phenotypes. Abbreviations: $\mathrm{AQP} 4-\mathrm{Ab}=$ aquaporin-4 antibody; MOG-Ab = myelin-oligodendrocyte glycoprotein antibody; $\mathrm{ON}=$ optic neuritis; $\mathrm{TM}=$ transverse myelitis; $\mathrm{ADEM}=$ acute disseminated encephalomyelitis

\section{Declaration of Competing Interest}

Dr. dos Passos reports scholarships from the European Committee for Treatment and Research in MS, World Federation of Neurology and Novartis; funding for research from Biogen, Novartis and Roche; travel grants from Merck, Roche, Sanofi-Genzyme and Teva; fees for editorial content from Bayer, Merck Serono and Roche; and compensation for advisory work from Biogen.

Dr. Elsone reports honorarium from Teva for developing an educational material.

Dr. Luppe has nothing to disclose with regards to this study.

Dr. Kitley reports she was supported by the NHS National Specialised Commissioning Group for Neuromyelitis Optica and has received travel grants from Biogen Idec, Novartis, Biogen, Roche, Merck and Teva, speaker honoraria from Novartis, Merck, Sanofi Genzyme and Terumo BCT and consultancy fees from Roche, Sanofi Genzyme and Merck.

Dr. Messina reports travel grants from Biogen, Novartis, Bayer, Merck, Almirall and honorarium for advisory work from Biogen.

Dr. Rodríguez Cruz has nothing to disclose with regards to this study.

Dr. Harding reports research funding from the MS Society of Canada and Novartis UK, speaker honoraria from Biogen and Merck, and support to attend educational meetings from Novartis.

Dr. Mutch has nothing to disclose with regards to this study.

Dr. Leite reports funding from NHS National Specialised Commissioning Group for Neuromyelitis optica, UK, and the NIHR Oxford Biomedical Research Centre, UK; and speaker honoraria or travel grants from Biogen Idec, Novartis, and the Guthy-Jackson Charitable Foundation.

Dr. Robertson reports support for scientific meetings and/or honorariums for lectures or advisory work from Biogen Idec, Celgene, No- vartis, Roche, Genzyme and CSL Behring; and grants from MS Society, Welcome Trust, NIHR, Novartis and Genzyme; he provides a regional service for patients with MS funded by Welsh Assembly Government.

Dr. Jacob reports compensation for advisory board, consulting, meeting attendance and speaking from Biogen, Terumo-BCT, Genentech, Shire and Chugai Pharmaceuticals.

Dr. Palace is partly funded by highly specialised services to run a national congenital myasthenia service and a neuromyelitis optica service. She has received support for scientific meetings and honorariums for advisory work from Merck Serono, Biogen Idec, Novartis, Teva, Chugai Pharma and Bayer Schering, Alexion, Roche, Genzyme, MedImmune, EuroImmun, MedDay, Abide and ARGENX, and grants from Merck Serono, Novartis, Biogen Idec, Teva, Abide, VielaBio and Bayer Schering. She has received grants from the MS Society, Guthy-Jackson Foundation, NIHR, Oxford Health Services Research Committee, EDEN, MRC, GMSI, and John Fell for research studies.

\section{Acknowledgements}

We are grateful to our colleagues providing the diagnostic assay for AQP4-Ab and MOG-Ab at the John Radcliffe Hospital (Angela Vincent, Patrick Waters and Mark Woodhall). We are also grateful to our Neurology and Ophthalmology colleagues across the UK for referring patients to the National Diagnostic and Advisory Service for Neuromyelitis Optica.

\section{References}

[1] D K Sato, D Callegaro, M A Lana-Peixoto, et al., Distinction between MOG antibodypositive and AQP4 antibody-positive NMO spectrum disorders, Neurology 82 (2014) 474-481, doi:10.1212/WNL.0000000000000101.

[2] J Kitley, P Waters, M Woodhall, et al., Neuromyelitis optica spectrum disorders with aquaporin-4 and myelin-oligodendrocyte glycoprotein antibodies: a comparative study, JAMA Neurol. 71 (2014) 1-8, doi:10.1001/ jamaneurol.2013.5857.

[3] S H M Hamid, D Whittam, M Saviour, et al., Seizures and encephalitis in myelin oligodendrocyte glycoprotein IgG disease vs aquaporin 4 IgG disease, JAMA Neurol. 75 (2018) 65, doi:10.1001/jamaneurol.2017.3196.

[4] K Fujihara, T Misu, I Nakashima, et al., Neuromyelitis optica should be classified as an astrocytopathic disease rather than a demyelinating disease, Clin. Exp. Neuroimmunol. 3 (2012) 58-73, doi:10.1111/j.1759-1961.2012.00030.x.

[5] K Kaneko, D K Sato, I Nakashima, et al., Myelin injury without astrocytopathy in neuroinflammatory disorders with MOG antibodies, J. Neurol. Neurosurg. Psychiatry (2016), doi:10.1136/jnnp-2015-312676.

[6] A Watad, S Azrielant, N L Bragazzi, et al., Seasonality and autoimmune diseases: the contribution of the four seasons to the mosaic of autoimmunity, $\mathrm{J}$. Autoimmun. 82 (2017) 13-30, doi:10.1016/j.jaut.2017.06.001.

[7] C Bowie, D Prothero, Finding causes of seasonal diseases using time series analysis, Int. J. Epidemiol. 10 (1981) 87-92, doi:10.1093/ije/10.1.87.

[8] K Harding, K Tilling, C Maciver, et al., Seasonal variation in multiple sclerosis relapse, J. Neurol. 264 (2017) 1059-1067, doi:10.1007/s00415-017-8485-0.

[9] D S Meier, K E Balashov, B Healy, et al., Seasonal prevalence of MS disease activity, Neurology 75 (2010) 799-806, doi:10.1212/WNL.0b013e3181f0734c.

[10] P M Rodríguez Cruz, L Matthews, M Boggild, et al., Time- and region-specific season of birth effects in multiple sclerosis in the United Kingdom, JAMA Neurol. 73 (2016) 954, doi:10.1001/jamaneurol.2016.1463.

[11] Y Jin, J de Pedro-Cuesta, M Söderström, et al., Seasonal patterns in optic neuritis and multiple sclerosis: a meta-analysis, J. Neurol. Sci. 181 (2000) 56-64.

[12] T Spelman, O Gray, M Trojano, et al., Seasonal variation of relapse rate in multiple sclerosis is latitude dependent, Ann. Neurol. 76 (2014) 880-890, doi:10.1002/ana.24287.

[13] P Waters, M Reindl, A Saiz, et al., Multicentre comparison of a diagnostic assay: aquaporin-4 antibodies in neuromyelitis optica, J. Neurol. Neurosurg. Psychiatry 87 (2016) 1005-1015, doi:10.1136/jnnp-2015-312601.

[14] J H Edwards, The recognition and estimation of cyclic trends, Ann. Hum. Genet. 25 (1961) 83-87.

[15] H Hakko, P Räsänen, J Tiihonen, P Nieminen, Use of statistical techniques in studies of suicide seasonality, 1970 to 1997, Suicide Life Threat. Behav. 32 (2002) 191-208.

[16] L S Freedman, The use of a Kolmogorov--Smirnov type statistic in testing hypotheses about seasonal variation, J. Epidemiol. Community Health 33 (1979) 223-228.

[17] S A Reijneveld, The choice of a statistic for testing hypotheses regarding seasonality, Am. J. Phys. Anthropol. 83 (1990) 181-184, doi:10.1002/ ajpa.1330830206.

[18] O Marrero, The performance of several statistical tests for seasonality in monthly data, J. Stat. Comput. Simul. 17 (1983) 275-296, doi:10.1080/ 00949658308810666. 
[19] J H Abramson, WINPEPI updated: computer programs for epidemiologists, and their teaching potential, Epidemiol. Perspect. Innov. 8 (2011) 1, doi:10.1186/ 1742-5573-8-1.

[20] R C Dale, C de Sousa, W K Chong, et al., Acute disseminated encephalomyelitis, multiphasic disseminated encephalomyelitis and multiple sclerosis in children, Brain 123 (Pt 12) (2000) 2407-2422.

[21] S N K Murthy, H S Faden, M E Cohen, R Bakshi, Acute disseminated encephalomyelitis in children, Pediatrics 110 (2002) e21-e21, doi:10.1542/ peds.110.2.e21.

[22] N Stewart, B Taylor, A-L Ponsonby, et al., The effect of season on cytokine expression in multiple sclerosis and healthy subjects, J. Neuroimmunol. 188 (2007) 181-186, doi:10.1016/j.jneuroim.2007.06.012.

[23] L P C Seguro, S G Pasoto, Seasonality and Autoimmunity. Infection and Autoimmunity, Elsevier, 2015, pp. 183-197.

[24] X C Dopico, M Evangelou, R C Ferreira, et al., Widespread seasonal gene expression reveals annual differences in human immunity and physiology, Nat. Commun. 6 (2015) 7000, doi:10.1038/ncomms8000.

[25] Y Oren, Y Shapira, N Agmon-Levin, et al., Vitamin D insufficiency in a sunny environment: a demographic and seasonal analysis, Isr. Med. Assoc. J. 12 (2010) 751-756.

[26] J-H Min, P Waters, A Vincent, et al., Low levels of vitamin D in neuromyelitis optica spectrum disorder: association with disease disability, PLoS One 9 (2014), doi:10.1371/journal.pone.0107274 e107274.
[27] M Muto, M Mori, Y Sato, et al., Seasonality of multiple sclerosis and neuromyelitis optica exacerbations in Japan, Mult. Scler. J. 19 (2013) 378-379, doi:10.1177/ 1352458512452332.

[28] D B Bichuetti, M O de Fragomeni, N A de Souza, Oliveira EML, Seasonality of 278 neuromyelitis optica relapses in a Brazilian cohort, Arq. Neuropsiquiatr. 73 (2015) 177-178, doi:10.1590/0004-282X20140213.

[29] J A D Leake, S Albani, A S Kao, et al., Acute disseminated encephalomyelitis in childhood: epidemiologic, clinical and laboratory features, Pediatr. Infect. Dis. J. 23 (2004) 756-764, doi:10.1097/01.inf.0000133048.75452.dd.

[30] C R Bamford, W A Sibley, C Thies, Seasonal variation of multiple sclerosis exacerbations in Arizona, Neurology 33 (1983) 697-697, doi:10.1212/ WNL.33.6.697.

[31] D E Goodkin, D Hertsgaard, Seasonal variation of multiple sclerosis exacerbations in North Dakota, Arch. Neurol. 46 (1989) 1015-1018, doi:10.1001/ archneur.1989.00520450085025.

[32] G Ogawa, H Mochizuki, M Kanzaki, et al., Seasonal variation of multiple sclerosis exacerbations in Japan, Neurol. Sci. 24 (2004) 417-419, doi:10.1007/ s10072-003-0200-5.

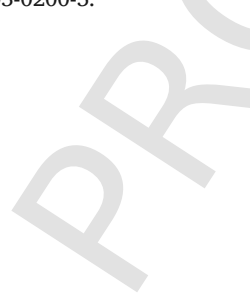

